\title{
A Synthetic Analog of Resveratrol Inhibits the Proangiogenic Response of Liver Sinusoidal Cells during Hepatic Metastasis
}

\author{
Elvira Olaso ${ }^{1, *}$, Aitor Benedicto ${ }^{1}$, Aritz Lopategi $^{1}$, Fernando P. Cossio ${ }^{2}$ and Beatriz Arteta ${ }^{1}$ \\ ${ }^{1}$ Tumor Microenvironment Group, Department of Cell Biology and Histology, School of Medicine and Dentistry, University of the \\ Basque Country, Leioa 48940, \\ ${ }^{2}$ Department of Organic Chemistry, School of Sciences, University of the Basque Country, Donosti 20018, Spain
}

\begin{abstract}
We utilized Fas21, a resveratrol analog, to modulate the function of hepatic stellate cells (HSCs) and liver sinusoidal endothelial cells (LSECs) during the angiogenic phase of murine liver metastasis by B16 melanoma and 51b colorectal carcinoma. Preangiogenic micrometastases were treated with Fas21 (1 mg/kg/day) or vehicle during the development of intra-angiogenic tracts. Mice treated with Fas21 showed reduced liver tumor foci in both liver metastasis models. Micrometastases were classified immunohistochemically, as well as according to their position coordinates and connection to local microvasculature. The volume of liver occupied by sinusoidal-type foci, containing infiltrating angiogenic capillaries, decreased by 50\% in Fas 21 -treated mice compared to vehicle-treated ones in both tumor metastasis models. The volume of portal foci, containing peripheral neoangiogenesis within a discontinuous layer of myofibroblasts, was similar in all experimental groups in both tumor metastasis models, but displayed enhanced necrotic central areas devoid of angiogenesis following Fas21 treatment. As a result, sinusoidal tumors from mice treated with Fas21 showed a 50\% reduction in desmin(+)/asma(+) HSCs and CD31(+) vessel density, and a 45\% reduction in intrametastatic VEGF mRNA compared with sinusoidal tumors from vehicle-treated mice. Necrotic portal metastases increased 2-4-fold in treated mice. In vitro, Fas21 reduced VEGF secretion by HSCs and 51b cells dose-dependently. Additionally, HSCs migration in response to tumor soluble factors was dose-dependently diminished by Fas 21 , as was LSEC migration in response to HSCs and tumor soluble factors. Resveratrol analog Fas21 inhibits the proangiogenic response of HSCs and LSECs during the development of murine liver metastasis.
\end{abstract}

Key Words: Hepatic stellate cell, Liver sinusoidal endothelial cell, Liver metastasis, Tumor microenvironment, Angiogenesis, Cancer

\section{INTRODUCTION}

Stromal myofibroblasts associated with epithelial tumors contribute substantially to the progression of cancer and constitute a promising new target for anticancer therapies (Mezawa and Orino, 2016). In the liver, tumor-activated hepatic stellate cells (HSCs) constitute a major element of the myofibroblastic component of the tumor stroma in primary (Makino et al., 2018) and metastatic (Shimizu et al., 2000) liver cancer. Moreover, the analysis of peritumoral myofibroblasts has prognostic relevance in human hepatocellular carcinoma (Barry et al., 2020). Activated HSCs contribute to liver metastasis via inflammatory and proangiogenic response-related mechanisms (Gulubova, 2004). Mechanistically, soluble factors derived from tumor cells induce HSCs' synthesis of pro- liferative factors for the tumor cells (Olaso et al., 1997) and soluble mediators such as VEGF, which elicit a proangiogenic response in the liver sinusoidal endothelium (Olaso et al., 2003). Both aspects are partially regulated by inducible cyclooxygenase 2 (Herrero et al., 2021).

Resveratrol is a nutraceutical compound with several antiinflammatory and anti-oxidative effects. Mechanistic and preclinical studies indicate that resveratrol prevents and delays malignant growth in experimental in vitro and in vivo assays (Kundu and Surh, 2004; Delmas et al., 2006) at relatively non-toxic doses. The pleiotropic activities of resveratrol are based on its ability to modulate multiple cell signaling molecules. In the liver, resveratrol inhibits experimental hepatic fibrosis, showing reduced collagen type I and lower density of $\alpha$-smooth muscle actin-expressing myofibroblasts (Lee et

\section{Open Access https://doi.org/10.4062/biomolther.2021.062}

This is an Open Access article distributed under the terms of the Creative Commons Attribution Non-Commercial License (http://creativecommons.org/licenses/by-nc/4.0/) which permits unrestricted non-commercial use, distribution, and reproduction in any medium, provided the original work is properly cited.
Received Apr 1, 2021 Revised Jul 13, 2021 Accepted Aug 14, 2021

Published Online Dec 7,2021

* Corresponding Author

E-mail: elvira.olaso@ehu.es

Tel: +34-946012880, Fax: +34-946013266 
al., 2010). We previously used B16-F10 melanoma (B16M) cells to study the effects of resveratrol treatment on the initial inflammatory step of hepatic metastasis. In this situation, resveratrol causes its effect primarily through the inhibition of IL18-dependent tumor cell adhesion to the hepatic endothelium (Salado et al., 2011).

Using resveratrol as a prototype, we synthesized $(E)-5-(((4-$ hydroxyphenyl)imino)methyl)benzene-1,3-diol, an unnatural resveratrol analog (Fas21) obtained in high yield by condensation between readily available reagents 4-aminophenol and 3,5-dihydroxybenzaldehyde (Cossio et al., 2006; Lu et al., 2012). We found that Fas21 interferes with metastases growth and with tumor neovessel formation during the angiogenic switch of the hepatic metastasis of murine B16M (Olaso et al., 2003) and 51b-Lim9 colon carcinoma (51bCC) (Solaun et al., 2002). Moreover, Fas21 downregulated the expression of intratumoral VEGF mRNA, which is intimately involved in angiogenesis. We also utilized an in vitro model composed of $\mathrm{B} 16 \mathrm{M}$ or $51 \mathrm{bCC}$ murine tumor cells, in conjunction with sinusoidal hepatic stellate cells (HSCs) and liver sinusoidal endothelial cells (LSECs), to study the dialogue between the tumor and the stroma microenvironment, along with the angiogenic development in murine metastasis formation in the liver. Our results showed that Fas21 remarkably inhibited metastasis of $\mathrm{B} 16 \mathrm{M}$ and $51 \mathrm{bCC}$ in the liver and reduced VEGF in situ. In vitro, Fas21 abrogated HSCs' and LSECs' chemotactic migration in response to soluble factors produced by the tumor microenvironment.

\section{MATERIALS AND METHODS}

\section{Chemical composition and computational analysis of Fas2 1 and resveratrol}

Fas21 was obtained as (E)-5-(((4-hydroxyphenyl)imino) methyl)benzene-1,3-diol in high yield by condensation between readily available reagents 4-aminophenol and 3,5-dihydroxybenzaldehyde. According to NMR analyses, the purity of the molecule was $>97 \%$ (Cushman et al., 2014). The structures of resveratrol and Fas21 were fully optimized within Density Functional Theory (DFT). The hybrid DFT functional used was B3LYP (Becke, 1993; Stephens et al., 1994) with a Grimme-Becke-Johnson (D3BJ) treatment of dispersion forces (Grimme et al., 2011). The split-valence 6-31G(d) basis set was used to describe hydrogen, carbon, and nitrogen atoms (Hehre et al., 1986). Partial charges were calculated within a natural bonding orbital (NBO) methodology (Weinhold, 2012).

\section{Hepatic metastasis}

Syngeneic C57BL/6J mice (male, 6-8 weeks old) were obtained from Charles River (Saint-Germain-sur-l'Arbresle, France). Housing, care, and experimental treatments were conducted following Directive 2010/63/EU and according to other institutional, national, and international guidelines regarding the protection and care of animals used for scientific purposes. Hepatic metastases were produced by intrasplenic injection into anesthetized C57 BL/6J mice $(n=30)$ of $3 \times 10^{5}$ viable B16 melanoma (B16M) cells or $3 \times 10^{5}$ viable $51 \mathrm{~b}$ coIon carcinoma $(51 \mathrm{bCC})$ cells. Five days after tumor cells injection, mice were divided into groups of five individuals that received $1 \mathrm{mg} / \mathrm{kg} /$ day Fas 21 or vehicle via intragastric tube. In one experiment, another group of mice was treated with resveratrol (1 mg/kg/day) via an intragastric tube. Mice were killed by cervical dislocation on the 12th day after the injection of tumor cells. Liver metastasis volume was calculated as the number of metastases per $100 \mathrm{~mm}^{3}$ of liver, based on the mean number of foci detected in three $10 \times 10 \mathrm{~mm}$ sections per liver (Solaun et al., 2002). Each experiment was carried out three times.

\section{Histochemistry}

Hematoxylin-eosin staining and $\alpha$-smooth muscle actin (SMA) expression analysis were performed on $4 \mu \mathrm{m}$-thick paraffin sections from zinc-fixed livers. In brief, slides were incubated for $1 \mathrm{~h}$ with a 1:50 dilution of SMA monoclonal antibody (DakoCytomation, Barcelona, Spain). Immunolabeled cells were detected with an avidin-biotin-peroxidase kit (Vecstatin ABC-AP kit; Vector Laboratories, CA, USA) according to the manufacturer's instructions. Sections ( $n=10$ per group) were sorted under microscopy observation according to their average diameter with a reference calibration scale, and the percentage of necrotic metastasis surface was determined. CD31/desmin double staining was performed on $14 \mu \mathrm{m}$ thick cryostat sections kept briefly in cold acetone $(n=10$, per group). Sections were sequentially incubated with anti-CD31 polyclonal antibody (BD Pharmigen, San Diego, CA, USA; overnight, 1:100 dilution), F(ab')2 rabbit anti-rat lgG-FITC (Serotec Ltd, Oxford, UK), 5\% normal rat serum, 3\% AffiniPure Fab rabbit anti-mouse IgG (1 h, 1:400, Jackson Labs, ME, USA), anti-human Desmin monoclonal antibody (2 h, 1:200 dilution, DakoCytomation), and rabbit anti-mouse IgG-Texas Red conjugate. All measurements were computer-assisted and performed at $20 \times$ magnification.

\section{Cell lines}

Murine B16M (B16-F10 subline) and murine $51 \mathrm{bCC}$ were obtained from the American Tissue Culture Collection (ATCC, LGC Standards S.L.U., Barcelona, Spain). The murine 51bCC subline Lim9 is a metastatic derivative from the parental cell line $51 \mathrm{bPT}$. This cell line was a kind gift from Dr. Robert S. Bresalier, who established it (Bresalier et al., 1987). Cells were maintained in DMEM supplemented with $10 \%$ FCS and were discarded after 10 passages.

\section{Isolation and primary culture of hepatic stellate cells and liver sinusoidal endothelial cells}

Mouse HSCs and LSECs were obtained as described elsewhere, with minor modifications (Smedsrød and Pertoft, 1985; Olaso et al., 2003; Arteta et al., 2010). Briefly, livers were perfused by a collagenase solution followed by a Percoll gradient. Cells were cultured in non-coated wells $\left(0.5 \times 10^{4}\right.$ cells/ $\mathrm{cm}^{2}$ ) in DMEM with $10 \%$ FCS, unless otherwise stated. All the cell cultures were at least $95 \%$ pure, as demonstrated by immunocytochemical analysis and Western blotting (Romayor et al., 2020).

\section{Generation of conditioned media}

Tumor cell-conditioned media was obtained from subconfluent cultures maintained overnight in serum-free DMEM. Then, the media was changed to serum-free DMEM for 24 h. HSC-conditioned media was obtained from sub-confluent cultures maintained for $8 \mathrm{~h}$ in DMEM. Tumor-activated HSCconditioned media was obtained from sub-confluent cultures maintained for $8 \mathrm{~h}$ in tumor cell-conditioned media diluted 1:1 
in DMEM. Cells were then pretreated, or not, for 30 min with Fas21; media was changed at least twice and maintained overnight in serum-free DMEM. All cell debris was removed by centrifugation and supernatants were stored at $-80^{\circ} \mathrm{C}$. All conditioned media was diluted 1:1 with fresh DMEM before use.

\section{Laser microdissection}

Laser microdissection was performed using an Arcturus ${ }^{\circledR}$ LCM system (Applied Biosystems Thermofisher, Pittsburg, PA, USA) on formalin-fixed, paraffin-embedded (FFPE) tissue samples. Tumor foci with an average $700 \mu \mathrm{m}$ diameter were collected and their RNA was amplified for standard quantitative real-time polymerase chain reaction $(n=20)$.

\section{Quantitative real-time polymerase chain reaction}

Quantitative real-time polymerase chain reaction (PCR) was performed in triplicate in $25 \mu \mathrm{L}$ with a SYBER Green PCR Core Reagent kit and a Gene Amp 5700 sequence detection system (all from PE Applied Biosystems, CA, USA), as previously described (Olaso et al., 2003). The following primers were used for murine VEGF: 5'GAGACCCTGGTGGACATC3' and 5'TTTCTTT- GGTCTGCATTC3' (this set of primers amplified the conserved region of all VEGF splicing forms); for murine b-actin, 5'GCCTTCCTTCTTGGGTATGG3' and 5'ACGCAGCTCAGTAACAGTCC $3^{\prime}$ were used. Each PCR run included 5 points of the standard curve (titrated dilutions of plasmid containing the specific fragment to evaluate). The target messenger was analyzed by measuring $\mathrm{Ct}$.

\section{VEGF measurement}

VEGF in non-concentrated cell culture supernatants was analyzed with a commercial enzyme-linked immunosorbent assay (ELISA) (R\&D Systems, Madrid, Spain) following the manufacturer's instructions.

\section{Cell migration assay}

HSCs $\left(3.5 \times 10^{4}\right)$ or LSECs $\left(3 \times 10^{5}\right)$ were seeded onto $0.001 \%$ type I collagen-coated inserts with $8 \mu \mathrm{m}$ pores and placed on top of $2 \mathrm{~cm}^{2}$ wells (BD Biosciences, San Jose, CA, USA) containing DMEM supplemented with $0.2 \%$ serum albumin, and were allowed to attach overnight. Then, media was replaced by DMEM containing $0.2 \%$ serum albumin in the upper compartment. The lower compartments were filled with either DMEM and $0.2 \%$ serum albumin (basal condition) or tumor-conditioned media in DMEM and $0.2 \%$ serum albumin. Some cells were pretreated with $10 \mu \mathrm{M}$ of Fas 21 or $10 \mu \mathrm{M}$ resveratrol $30 \mathrm{~min}$ before the addition of the chemoattractant to the lower compartment. After 8-24 h, non-migrated cells were removed and migrated cells were fixed, stained with hematoxylin-eosin, and counted using a light microscope (20x) in 5 random fields per well.

\section{Statistics}

Results refer to mean \pm SD. Individual comparisons were made with Student's two-tailed, unpaired $t$-test. The criterion for significance was $p<0.05$ for all comparisons. In vivo experiments were repeated twice. In vitro experiments were performed at least in duplicate with two independent samples.

\section{RESULTS}

\section{Fas 21 is a geometric and stereo-electronic analog of resveratrol}

Fully optimized structures of resveratrol and Fas21 (Fig. 1) show similar structures for both compounds. The lowest conformation of resveratrol shows a coplanar structure, as is shown by the ca. 180 deg. dihedral angles formed by the central trans $\mathrm{HC}=\mathrm{CH}$ moiety and both $\mathrm{A}$ and $\mathrm{B}$ phenyl groups. The lowest energy conformation of Fas 21 showed a slightly distorted structure, with a departure from the coplanarity of dihedral $\mathrm{HC}=\mathrm{N}-\mathrm{B}$ angle. However, the low energy profile associated with the rotation of both aryl groups shows a similar average structure. This is shown by the distances among the three hydroxyl groups, which are very similar for resveratrol and Fas21. The solvent accessible surface areas (SASA) computed for both compounds are also very similar, though Fas21 is more polar than resveratrol, as is shown by the slightly higher polar surface area (PSA) computed for Fas21. This slightly higher polarity is induced by the negative partial charge of the $\mathrm{sp}^{2}$-hybridized nitrogen atom of this latter compound. In summary, the substitution of $\mathrm{CH}$ moiety in resveratrol by an $\mathrm{N}\left(\mathrm{sp}^{2}\right)$ atom in Fas21 results in a very similar compound whose chemical synthesis is simpler. Therefore, this synthetic molecule is suitable for further biological studies.

\section{Fas21 reduces metastatic growth of intrasplenically injected B16 Melanoma and 51b Colon Carcinoma cells}

Mice were injected intrasplenically with syngenic B16M or $51 \mathrm{bCC}$ cells. After five days, small avascular tumor foci (less than $250 \mu \mathrm{m}$ in diameter) were evident in the liver (Olaso et al., 1997). At that time, mice received $1 \mathrm{mg} / \mathrm{kg} /$ day Fas 21 or vehicle intragastrically, and their livers were analyzed seven days afterward. All treatments were well tolerated, and treated mice did not suffer body weight loss or any apparent lifethreatening toxicity compared to control mice receiving the same volume of vehicle.

Treatment with Fas21 reduced the overall volume of liver metastasis, independent of the tumor cell line used. Small avascular foci with a diameter below $0.4 \mathrm{~mm}$ were not affected by the treatment, while differences between treated and untreated mice were significant for neoangiogenic tumor foci larger than $0.4 \mathrm{~mm}$ in diameter, where the average reduction was $\sim 50 \%$ compared to the respective untreated control mice (Fig. 2). The largest inhibition took place in the panlobular micrometastasis group of B16M-metastasized mice, where Fas21 reduced tumor foci diameter by up to five-fold (Fig. 2A). A similar effect was observed in $51 \mathrm{bCC}$. Fas 21 reduced by $\sim$ two-fold the number of micrometastases larger than $0.4 \mathrm{~mm}$ in diameter (Fig. 2B).

\section{Fas21 inhibits B16 Melanoma and 51b Colon Carcinoma hepatic metastases of Sinusoidal Type}

Liver sections were stained for smooth alpha-actin (SMA) expression to analyze the effect of Fas21 on the angiogenic pattern of B16M and $51 \mathrm{bCC}$ micrometastases, according to their myofibroblastic support (Olaso et al., 2003, Solaun et al., 2002). As shown in Fig. 3, 55-61\% of vehicle-treated metastases larger than $250 \mu \mathrm{m}$ belonged to the sinusoidal-type-or intratumoral containing a network of sinusoidal-like capillaries-while $28-36 \%$ of metastases were of portal- or peritumoral-type located in the vicinity of portal tracts and surrounded 
A
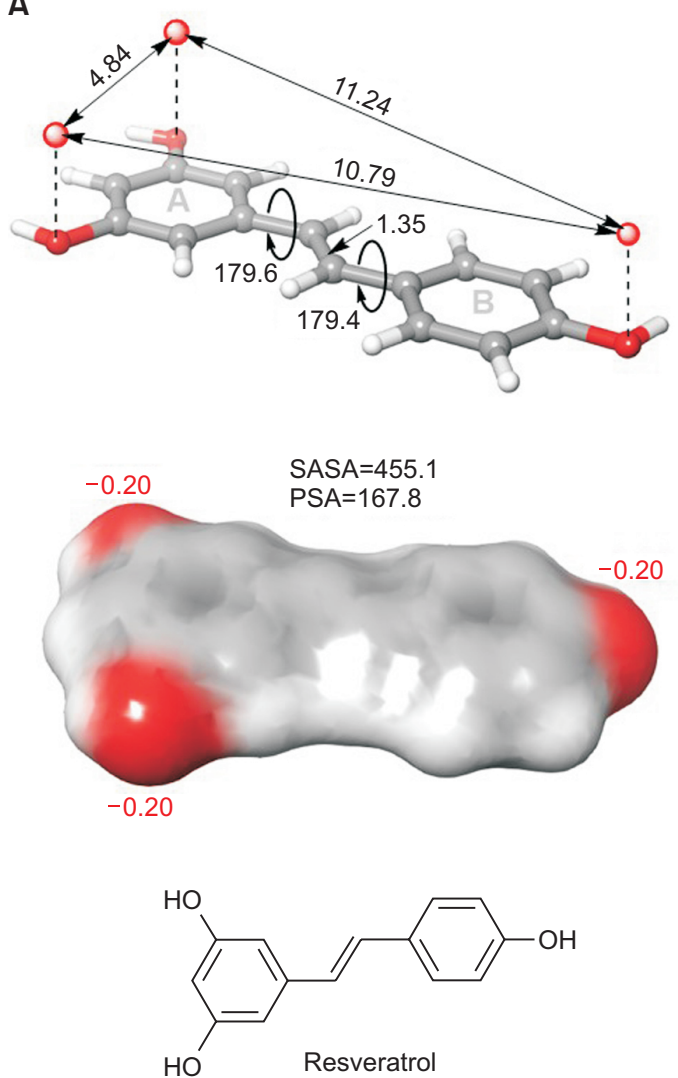

B
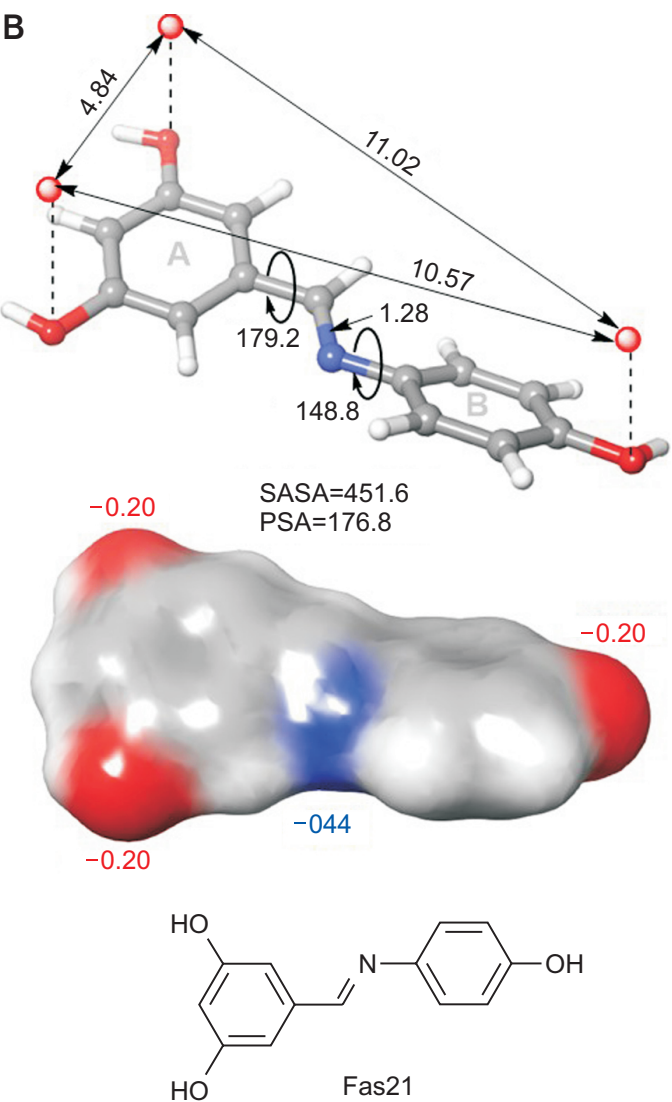

Fig. 1. Structural and electronic features of Fas21. Geometric parameters, partial charges, and solvent accessible surfaces of (A) resveratrol and (B) Fas21. Bond distances are given in A. Dihedral angles of the central moieties $(\mathrm{HC}=\mathrm{CH}$ and $\mathrm{HC}=\mathrm{N}$, respectively) with phenyl groups $A$ and $B$ are given in absolute value and deg. Solvent accessible surface areas (SASA) and polar surface areas (PSA) have been calculated with a probe radius of $1.4 \AA$ (associated with water) and are given in $\AA^{2}$. Partial charges in the hydroxyl groups (in red) and the $\mathrm{sp}^{2}$-hybridized nitrogen atom (in blue) are given in atomic units.
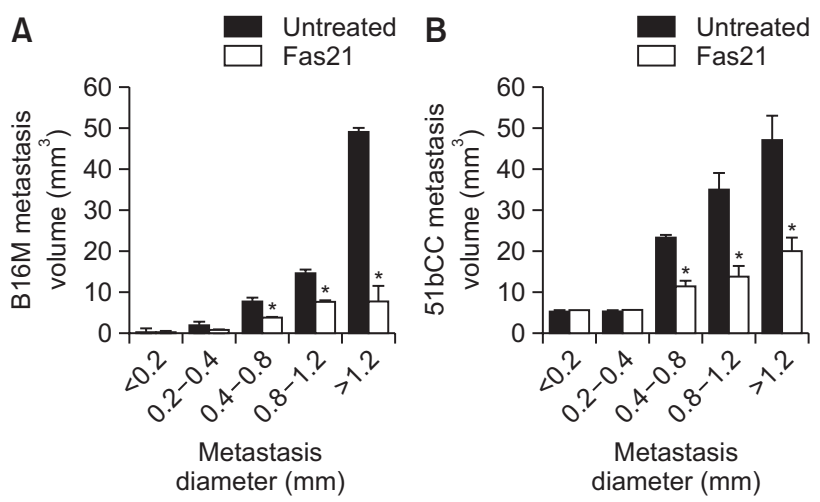

Fig. 2. Effect of Fas 21 administration on avascular micrometastases from B16M and 51bCC cells. Five days after cancer cell injection, Fas21 (1 mg/kg/day) or vehicles were administered via an intragastric tube for the following 7 days. Then, livers were processed for histological analyses. Metastatic foci were arbitrarily subdivided into groups attending to their average diameter, and the average density of each group was calculated in liver sections from Fas21-treated or vehicle-treated (untreated) animals. (A) B16M, (B) $51 \mathrm{bCC}$. Histograms represent the average values of two independent experiments $(n=30)$. ${ }^{*} p<0.01$ with respect to vehicle-injected mice. F21: Fas21. by a discontinuous layer of SMA-positive myofibroblasts. The remaining $7-11 \%$ foci were avascular ones and did not contain SMA. Fas 21 reduced sinusoidal-type metastasis density by $\sim 54 \%$ in the B16M model (Fig. 3E), and by $-62 \%$ in the $51 \mathrm{~b}-$ CC model (Fig. 3F). The density of portal-type metastases was not significantly modified, but their average size was larger in Fas21-treated B16M foci tumors than in their respective controls (data not shown). In both models, Fas21 promoted central necrosis (Supplementary Fig. 1). In the B16M model, the portal metastasis necrosis was two-fold higher in treated mice than untreated ones (Supplementary Fig. 1A). In the $51 \mathrm{bCC}$ model, which was less prone to necrosis, the effect of Fas21 was more intense and reached a four-fold increase in metastases of a portal-type larger than $0.8 \mathrm{~mm}$ in diameter of treated mice compared to untreated ones (Fig. 3D).

Fas21 Inhibits angiogenic vessel formation in sinusoidal-
type metastasis of B16M and reduces in vitro migration
of Hepatic Stellate Cells and Liver Sinusoidal Endothelial
Cells
The recruitment of CD31-expressing angiogenic endothelial cells, associated with SMA/desmin-expressing tumor-activated HSCs in the development of avascular metastases, is a key step for tumor angiogenesis in hepatic micrometastases (Olaso et al., 2003). Moreover, tumor-derived factors promote 


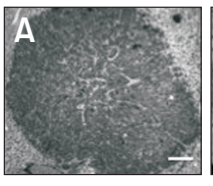

$\mathbf{E}$

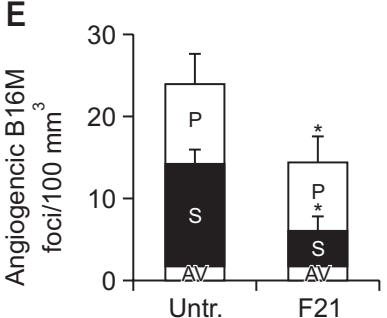

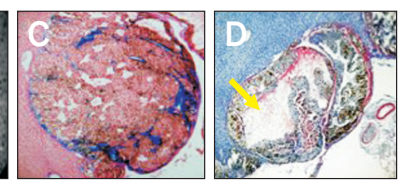

$\mathrm{F}$

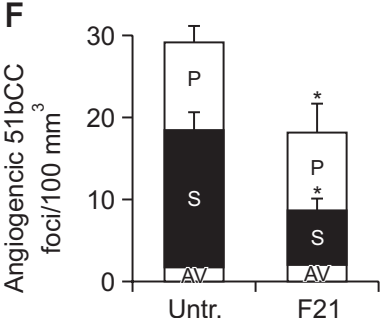

Fig. 3. Effect of Fas21 on the occurrence of B16M or 51bCC micrometastasis types regarding their position coordinates in liver tissue and their connection to local microvasculature. Metastatic foci in their angiogenic stage, larger than $250 \mu \mathrm{m}$ in diameter and stained with $\mathrm{H} / \mathrm{E}$ or anti-SMA antibodies, were separated according to their sinusoidal-type or portal-type character, were counted under microscopy, and are expressed as foci per $100 \mathrm{~mm}^{3}$ : (A) Sinusoidal-type $51 \mathrm{bCC}$ micrometastasis from untreated mouse associated with infiltrating capillaries. (B) Portal-type 51bCC micrometastasis from untreated mouse containing blood-filled caveoli. (C) Sinusoidal-type B16M micrometastasis from Fas21 mouse containing SMA-positive myofibroblasts (blue staining) associated with infiltrating angiogenic capillaries. (D) Portal-type B16M micrometastasis from Fas21-treated mice surrounded by a discontinuous layer of SMA-positive myofibroblasts (red staining) containing blood-filled caveoli. ( $E, F$ ) Histograms on the number of sinusoidal and portal foci in B16M and $51 \mathrm{bCC}$ metastasized livers. P: portal-type metastatic foci; S: sinusoidal-type metastatic foci; AV: avascular metastatic foci; F21: Fas21. Scale bar: $100 \mu \mathrm{m}$. Yellow arrows: blood-filled caveoli. * $p<0.05$ versus untreated mice.

HSCs migration in the hepatic metastasis microenvironment. In turn, tumor-activated HSCs induce LSEC proangiogenic migration (Olaso et al., 2003, Herrero et al., 2021). Immunohistological analyses of intratumoral cellular composition in B16M metastatic foci showed angiogenic vessels formed by CD31-expressing cells in close contact with SMA/desminexpressing cells (Fig. 4A-4D). Fas21 significantly reduced the density of CD31-expressing angiogenic vessels by $55 \%$ and desmin-expressing HSCs by $50 \%$ (Fig. 4E, 4F) compared to untreated mice. Moreover, Fas21 administration caused the impaired formation of vessels longer than $120 \mu \mathrm{m}$ compared to those observed in metastases of untreated mice (Fig. 4G).

In vitro, B16M-conditioned media induced untreated HSCs migration compared to basal media. Such activation was inhibited dose-dependently by increasing concentrations of Fas21 (Fig. 5A). B16M-derived factors and HSCs-derived factors are two main promotors of LSECs' migration in the hepatic metastasis microenvironment. In vitro, freshly isolated LSECs pretreated with $10 \mu \mathrm{M}$ of Fas 21 migrated significantly less than LSECs maintained in the presence of vehicle, in response to both B16M- and HSCs-conditioned media (Fig. 5B). Under those experimental conditions, there was no observed apoptosis or cell toxicity, while Fas 21 higher than $25 \mu \mathrm{M}$ promoted cell detachment (data not shown).
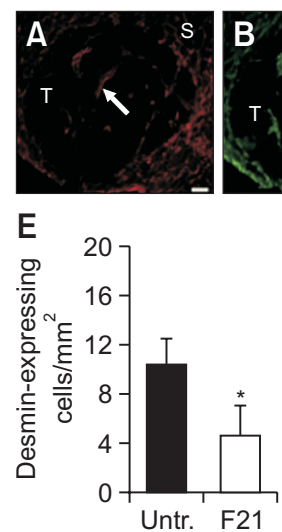

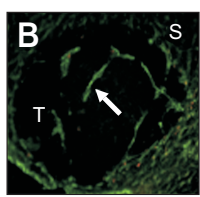

F
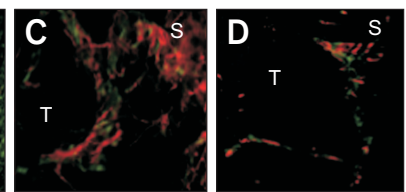

G
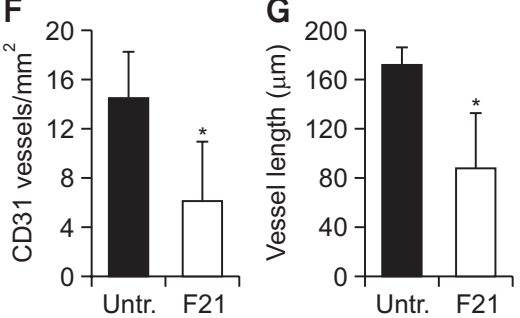

Fig. 4. Effect of Fas 21 in intratumoral desmin expression and angiogenesis of B16M micrometastasis. Cryostat sections from B16M-bearing livers stained with anti-desmin (in red) and/or antiCD31 (in green) antibodies: $(A, B)$ desmin-expressing cells $(A)$ or CD-31-expressing cells in non-necrotic tumor foci. (C, D) Intratumoral neoangiogenic tracts composed of CD31-expressing endothelial cells and associated desmin-expressing pericytes are connected to liver sinusoidal capillaries. (E-G) Density and length of desmin- or CD31-expressing vessel tracts in non-necrotic micrometastasis. F21: Fas21. T: tumor foci. S: hepatic sinusoids. White arrows: representative microvessel inside the tumor foci. Scale bar: $100 \mu \mathrm{m} .{ }^{*} p<0.05$ versus untreated mice.
A

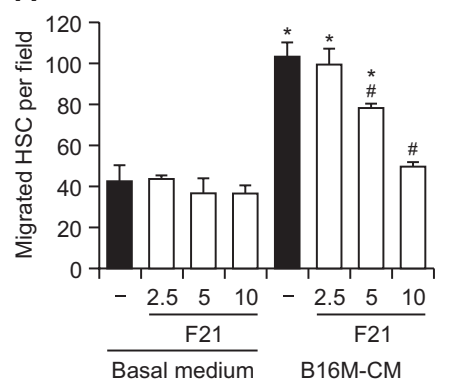

B

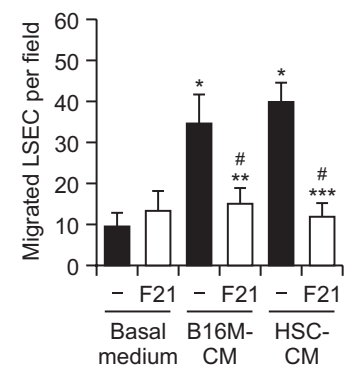

Fig. 5. Effect of Fas21 on LSECs' chemotactic migration induced by B16M cells and by tumor-activated HSCs: (A) HSCs were cultured on collagen-coated inserts in the presence of increasing concentrations of Fas21. Then, media was replaced by DMEM in the cell compartment and supernatants from B16M in the lower compartment. Migrated cells were stained with $\mathrm{H} / \mathrm{E}$ and counted under the microscope. ${ }^{*} p<0.05$ versus control cells in basal medium number of migrated HSCs in basal medium: $15 \pm 8$ and ${ }^{*} p<0.05$ versus control cells in B16M-CM. (B) LSECs were cultured on collagen-coated inserts and either $10 \mu \mathrm{M}$ of Fas 21 or vehicle. Then, media was changed and replaced by basal media in the upper cell compartment and supernatants from B16M-treated HSCs in the lower compartment. Migrated cells were stained with $\mathrm{H} / \mathrm{E}$ and counted under the microscope. ${ }^{*} p<0.05,{ }^{* *} p<0.01$ and ${ }^{* * *} p<0.005$ versus control LSECs in basal medium (10 \pm 2 LSECs) and $\# p<0.05$ versus control cells in B16M-CM. F21: Fas21.

\section{Fas21 reduces intratumoral VEGF mRNA levels in 51b Colon Carcinoma hepatic metastasis and VEGF secretion by cultured $51 \mathrm{~b}$ Colon Carcinoma cells and tumor- activated Hepatic Stellate Cells}

VEGF is the main angiogenic factor in liver metastasis. Tumor cells and activated HSCs are major cellular sources of 
VEGF in the metastasized liver. Laser microdisectates from angiogenic, non-necrotic, $51 \mathrm{bCC}$ tumor foci (Fig. 3A) contained an average of two-fold more VEGF than close tissue
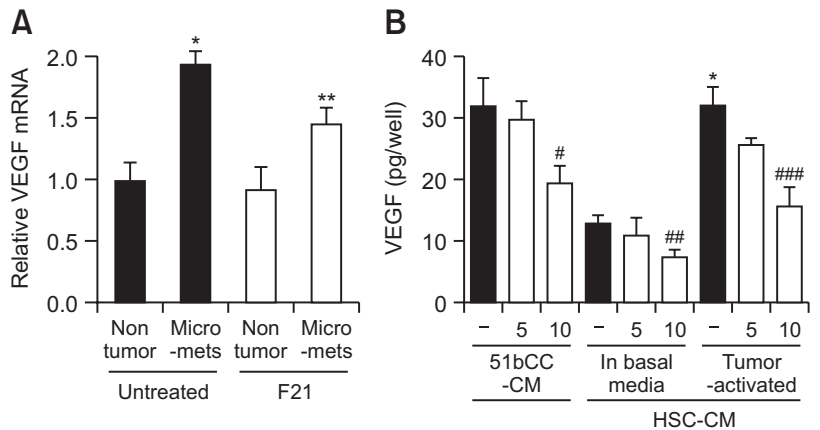

Fig. 6. Fas21 reduces VEGF in the tumor microenvironment: (A) Quantitative RT-PCR analysis of VEGF mRNA in laser microdissected foci from murine liver metastasis by $51 \mathrm{bCC}$. =VEGFCt $/$ Bactin $\mathrm{Ct}=35,71 / 34,70$. (B) In vitro ELISA measurement of VEGF in $51 \mathrm{bCC}$ - and HSC-conditioned media after treatment or not with 10 $\mu \mathrm{M}$ of Fas21. ${ }^{*} p<0.05$ versus non-tumoral tissue. ${ }^{* *} p<0.05$ versus micrometastases of $51 \mathrm{bCC}$-bearing mice treated with Fas 21. $\# p<0.05$ versus untreated $51 \mathrm{bCC}$ cells. ${ }^{\#} p<0.05$ versus untreated basal HSCs. ${ }^{\# \#} p<0.05$ versus untreated tumor-activated HSCs. that was unaffected by the tumor (Fig. 6A). Hepatic metastases of mice treated with Fas 21 contained $\sim 45 \%$ less VEGF mRNA than those from untreated mice. Interestingly, Fas21 did not affect VEGF mRNA levels in non-tumoral liver tissue. Finally, ELISA showed that Fas21 dose-dependently reduced the amount of VEGF secreted by cultured $51 \mathrm{bCC}$ (Fig. 6B). Moreover, $10 \mu \mathrm{M}$ of Fas 21 inhibited $50 \%$ of VEGF secreted by freshly isolated, quiescent HSCs and the induction of VEGF production generated by HSC activation through exposure to $51 \mathrm{bCC}$ supernatants (Fig. 6B). Similar results were obtained at the mRNA level (Supplementary Fig. 2). Semiquantitative RTPCR showed that treatment with $10 \mu \mathrm{M}$ of Fas21 significantly reduced the amount of VEGF mRNA synthesized by HSCs activated by either B16M- or 51bCC-conditioned media.

On the whole, these data demonstrate the possible role of Fas21 in relation to metastasis on at least at two levels: first, on the tumor cells, and second, on the generation of a prometastatic milieu that impairs proangiogenic stromal cell reaction.

\section{Fas21 efficiency as a liver metastasis inhibitor compared to resveratrol}

We previously demonstrated that resveratrol inhibits experimental hepatic metastasis by intrasplenic injection of B16M (Salado et al., 2011). To compare the inhibitory ability of Fas21 with that of resveratrol against the metastatic development of
A
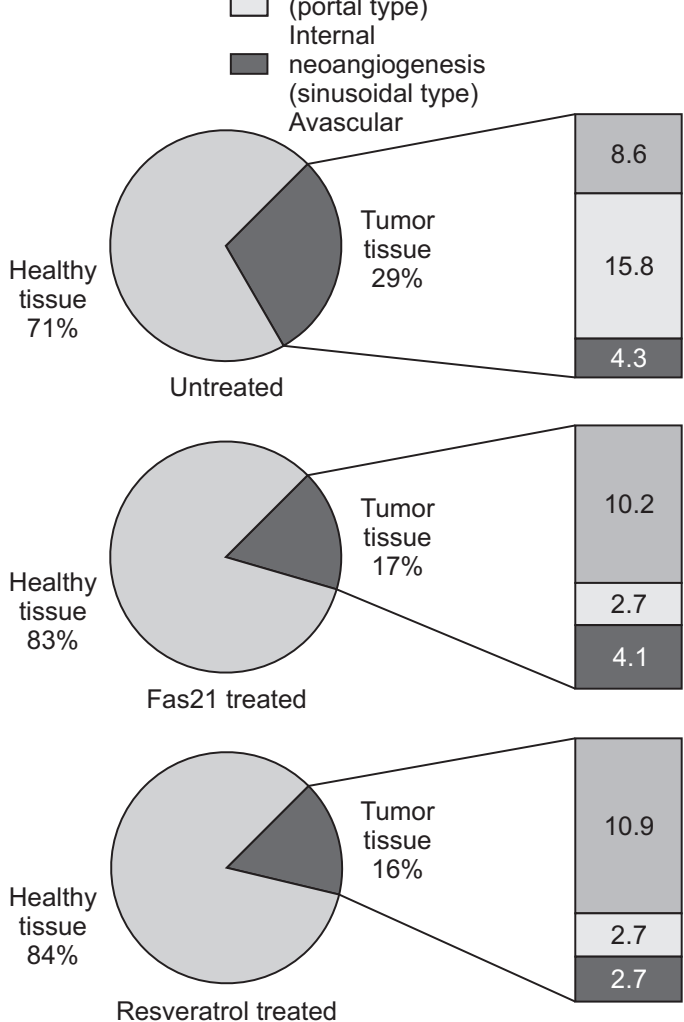

B

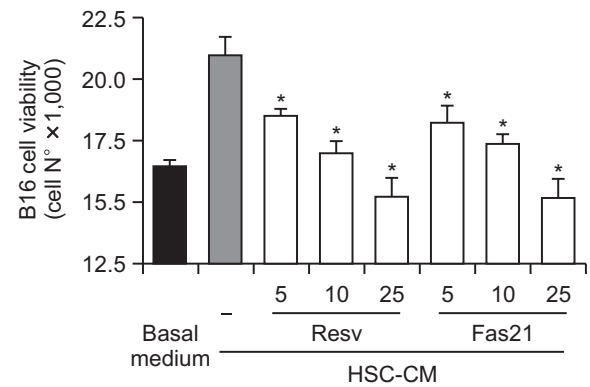

C

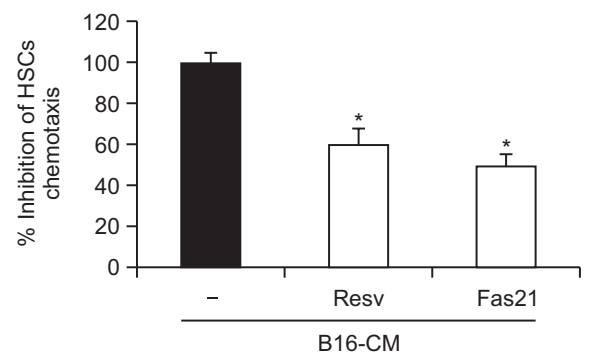

Fig. 7. Comparison of the antimetastatic effects on B16M between Fas21 and resveratrol: (A) Mice were injected intrasplenically with B16M cells. Animals were treated intragastrically with $1 \mathrm{mg} / \mathrm{kg} /$ day of Fas 21 , resveratrol, or vehicle. On day 12, livers were analyzed immunohistochemically. (B) Comparison of the effects of Fas21 and resveratrol on the proliferative potential of HSC on B16 cells. Tumor cells were culture in the presence of HSCs soluble factors pre-treated with either resveratrol or Fas 21. Viability was measured after $48 \mathrm{~h}$ by Presto Blue. ${ }^{*} p<0.01$ with respect to B16 viability cultured in the presence of untreated HSCs. 
B16M in the liver, mice received Fas21, resveratrol (both intragastrically at a dose of $1 \mathrm{mg} / \mathrm{kg} /$ day), or vehicle. Fig. 7A shows that the inhibition of metastatic development, measured using immunohistochemical parameters, was similar using both compounds. Additionally, growing concentrations ranging from 5 to $25 \mu \mathrm{M}$ of either compound produced a similar inhibitory effect on B16 proliferation in response to HSCs soluble factors (Fig. 7B). Moreover, a concentration of $10 \mu \mathrm{M}$ of either compound inhibited HSCs migration in response to B16 soluble factors (Fig. 7C).

\section{DISCUSSION}

In the present work, we used an experimental model of liver metastasis where tumor growth and the development of an intratumoral angiogenic stroma depended on the reciprocal interaction between host cells and tumor cells, and the resulting angiogenic response is partly regulated by VEGF. In this model, we analyzed the effect of Fas21, a resveratrol analog, on the angiogenic response of liver sinusoidal endothelial cells and hepatic stellate cells to metastasis development by B16 melanoma and 51b colon carcinoma.

Livers from mice treated with Fas 21 contained significantly fewer metastasis foci than untreated ones. This was evident in the small set of foci, which may indicate that micrometastasis regression was taking place on a fraction of the metastases that were developed before drug administration. Additionally, sinusoidal-type growth was highly inhibited by Fas21, but not portal-type growth. These may suggest that, in this model, Fas21 exerts its effects at two levels: one, directly to the tumor cells, and two, on the tumor-associated stroma, being largely more effective on sinusoidal-type metastasis. This hypothesis is supported by our in vitro results, where Fas 21 prevented the migratory response of HSCs and LSEC exerted by tumor cells and HSCs supernatants, respectively. As previously reported by us and others, HSCs and LSECs conform the cellular supply for neoangiogenesis in experimental murine metastasis to the liver (Olaso et al., 2003). Once activated by the tumor microenvironment, HSCs/LSECs dialogue results in the synthesis of a proangiogenic stroma that favors metastatic growth (Vidal-Vanaclocha, 2008). Our results show that Fas21 reduces HSCs and LSECs' proangiogenic, invasive, and secretory responses by an average of $50 \%$, indicating that Fas 21 deserves further analysis as a coadjuvant candidate in metastatic treatment.

We previously found that resveratrol alters the inflammation phase that occurs during B16M colonization of the liver through inhibition of IL-18-dependent expression of VCAM-1 by tumor-activated LSECs and adhesion- and proliferationstimulating effects of IL-18 on metastatic B16 cells through hydrogen peroxide-dependent NF-kB translocation blockade (Salado et al., 2011). In this work, we analyze the angiogenic phase that occurs once tumor cells have initiated the formation of micrometastases. Our findings indicate that the resveratrol analog Fas21 reduces VEGF production in the tumor microenvironment at this metastatic stage produced by both B16 and $51 \mathrm{bCC}$ cells. Our work corroborates previous studies that demonstrate how resveratrol suppresses the growth of new blood vessels in developing embryos (Bråkenhielm et al., 2001) and blunts subcutaneous C26 colon carcinoma vascularization (Walter et al., 2008).
Our findings suggest that the antitumor effects of Fas21 could be explained by its indirect anti-stromal activities as an inhibitor of the proangiogenic milieu generated by tumor infiltration of the hepatic sinusoids, as well as by its direct effect on tumor cell growth.

\section{CONFLICT OF INTEREST}

FPC is a consultant of Quimatryx SL.

\section{ACKNOWLEDGMENTS}

This work was supported in part by a grant from the Spanish Ministry of Health (04/2785) to EO.

\section{REFERENCES}

Arteta, B., Lasuen, N., Lopategi, A., Sveinbjörnsson, B., Smedsrød, B. and Vidal-Vanaclocha, F. (2010) Colon carcinoma cell interaction with liver sinusoidal endothelium inhibits organ-specific antitumor immunity through interleukin-1-induced mannose receptor in mice. Hepatology 51, 2172-2182.

Barry, A. E., Baldeosingh, R., Lamm, R., Patel, K., Zhang, K., Dominguez, D. A., Kirton, K. J., Shah, A. P. and Dang, H. (2020) Hepatic stellate cells and hepatocarcinogenesis. Front. Cell Dev. Biol. 8, 709.

Becke, A. D. (1993) Density-functional thermochemistry. III. The role of exact exchange. J. Chem. Phys. 98, 5648.

Bråkenhielm, E., Cao, R. and Cao, Y. (2001) Suppression of angiogenesis, tumor growth, and wound healing by resveratrol, a natural compound in red wine and grapes. FASEB J. 15, 1798-1800.

Bresalier, R. S., Hujanes, E. S., Raper, S. E., Roll, F. J., Itzkowitz, S. H., Martin, G. R. and Kim, Y. S. (1987) An animal model for colon cancer metastasis: establishment and characterization of murine cell lines with enhanced liver-metastasizing ability. Cancer Res. 47, 1398-1406.

Cossio, F., Aldaba, E., Vara, Y., Zubia, A., Vivanco, S., Mendoza, L., Salado, C., Gallot, N. and Vidal-Banaclocha, F. (2006) New nitrogen-containing trans-stilbene analogs and their preparation, antimetastatic activity and their medical applications. United States Patent US 20,110,060,038. 2006 Oct 19.

Cushman, M., Georg, G. I., Holzgrabe, U. and Wang, S. (2014) Absolute quantitative ${ }^{1} \mathrm{H}$ NMR spectroscopy for compound purity determination. J. Med. Chem. 57, 9219.

Delmas, D., Lancon, A., Colin, D., Jannin, B. and Latruffe, N. (2006) Resveratrol as a chemopreventive agent: a promising molecule for fighting cancer. Curr. Drug Targets 7, 423-442

Grimme, S., Ehrlich, S. and Goerigk, L. (2011) Effect of the damping function in dispersion corrected density functional theory. J. Comput. Chem. 32, 1456-1465.

Gulubova, M. V. (2004) Collagen type IV, laminin, alpha-smooth muscle actin (alphaSMA), alpha1 and alpha6 integrins expression in the liver with metastases from malignant gastrointestinal tumours. Clin. Exp. Metastasis 21, 485-494.

Hehre, W. J., Radom, L., Schleyer, P. R. and Pople, J. A. (1986) AB INITIO Molecular Orbital Theory. Wiley, New York.

Herrero, A., Benedicto, A., Romayor, I., Olaso, E. and Arteta, B. (2021) Inhibition of COX-2 impairs colon cancer liver metastasis through reduced stromal cell reaction. Biomol. Ther. (Seoul) 29, 342-351.

Kundu, J. K. and Surh, Y. J. (2004) Molecular basis of chemoprevention by Resveratrol: NF-kappaB and AP-1 as potential targets. Mutat. Res. 555, 65-80.

Lee, E. S., Shin, M. O., Yoon, S. and Moon, J. O. (2010) Resveratrol inhibits dimethylnitrosamine-induced hepatic fibrosis in rats. Arch. Pharm. Res. 33, 925-932.

Lu, J., Li, C., Chai, Y. F., Yang, D. Y. and Sun, C. R. (2012) The anti- 
oxidant effect of imine resveratrol analogues. Bioorg. Med. Chem. Lett. 22, 5744-5747.

Makino, Y., Hikita, H., Kodama, T., Shigekawa, M., Yamada, R., Sakamori, R., Eguchi, H., Morii, E., Yokoi, H., Mukoyama, M., Hiroshi, S., Tatsumi, T. and Takehara, T. (2018) CTGF mediates tumorstroma interactions between hepatoma cells and hepatic stellate cells to accelerate HCC progression. Cancer Res. 78, 4902-4914.

Mezawa, Y and Orino, A. (2016) The roles of tumor- and metastasispromoting carcinoma-associated fibroblasts in human carcinomas. Cell Tissue Res. 365, 675-689.

Olaso, E., Santisteban, A., Bidaurrazaga, J., Gressner, A. M., Rosenbaum, J. and Vidal-Vanaclocha, F. (1997) Tumor-dependent activation of rodent hepatic stellate cells during experimental melanoma metastasis. Hepatology 26, 634-642.

Olaso, E., Salado, C., Egilegor, E., Gutierrez, V., Santisteban, A., SanchoBru, P., Friedman, S. L. and Vidal-Vanaclocha, F. (2003) Proangiogenic role of tumor-activated hepatic stellate cells in experimental melanoma metastasis. Hepatology 37, 674-685.

Romayor, I., Badiola, I., Benedicto, A., Marquez, J., Herrero, A., Arteta, B. and Olaso, E. (2020) Silencing of sinusoidal DDR1 reduces murine liver metastasis by colon carcinoma. Sci. Rep. 10, 18398.

Salado, C., Olaso, E., Gallot, N., Valcarcel, M., Egilegor, E., Mendoza, L. and Vidal-Vanaclocha, F. (2011) Resveratrol prevents inflammation-dependent hepatic melanoma metastasis by inhibiting the secretion and effects of interleukin-18. J. Transl. Med. 9, 59.

Shimizu, S., Yamada, N., Sawada, T., Ikeda, K., Kawada, N., Seki, S.,
Kaneda, K. and Hirakawa, K. (2000) In vivo and in vitro interactions between human colon carcinoma cells and hepatic stellate cells. Jpn. J. Cancer Res. 91, 1285-1295.

Solaun, M. S., Mendoza, L., De Luca, M., Gutierrez, V., Lopez, M. P., Olaso, E., Sim, B. K. L. and Vidal-Vanaclocha, F. (2002) Endostatin inhibits murine colon carcinoma sinusoidal-type metastases by preferential targeting of hepatic sinusoidal endothelium. Hepatology 35, 1104-1116.

Smedsrød, B. and Pertoft, H. (1985) Preparation of pure hepatocytes and reticuloendothelial cells in high yield from a single rat liver by means of Percoll centrifugation and selective adherence. J. Leukoc. Biol. 38, 213-230.

Stephens, P. J., Deviln, F. J., Chabalowski, C. F. and Frisch, M. J. (1994) Ab initio calculation of vibrational absorption and circular dichroism spectra using density functional force fields. J. Phys. Chem. 98, 11623-11627.

Vidal-Vanaclocha, F. (2008) The prometastatic microenvironment of the liver. Cancer Microenviron. 1, 113-129.

Walter, A., Etienne-Selloum, N., Brasse, D., Khallouf, H., Bronner, C., Rio, M. C., Beretz, A. and Schini-Kerth, V. B. (2008) Intake of grape-derived polyphenols reduces $\mathrm{C} 26$ tumor growth by inhibiting angiogenesis and inducing apoptosis. FASEB J. 24, 3360-3369.

Weinhold, F. (2012) Natural bond orbital analysis: a critical overview of relationships to alternative bonding perspectives. J. Comput. Chem. 33, 2363-2379. 